\title{
Resting-state magnetoencephalography study of "small world" characteristics and cognitive dysfunction in patients with glioma
}

This article was published in the following Dove Press journal:

OncoTargets and Therapy

2 April 2013

Number of times this article has been viewed

\author{
Xin-Hua Hu \\ Ting Lei \\ Hua-Zhong Xu \\ Yuan-Jie Zou \\ Hong-Yi Liu
}

Department of Neurosurgery, Brain Hospital Affiliated to Nanjing Medical University, Nanjing, People's Republic of China
Correspondence: Xin-Hua Hu, Department of Neurosurgery, Brain Hospital Affiliated to Nanjing Medical University, Nanjing 210029,

People's Republic of China

$\mathrm{Tel}+862582296423$

Fax +86 25 837I 9457

Email huxinhua200I@yahoo.cn

Hong-Yi Liu

Department of Neurosurgery, Brain Hospital Affiliated to Nanjing Medical University, Nanjing 210029 ,

People's Republic of China

Tel +862582296423

Fax +8625837/9457

Email liuhybh@yahoo.cn
Background: The purpose of this study was to analyze "small world" characteristics in glioma patients in order to understand the relationship between cognitive dysfunction and brain functional connectivity network in the resting state.

Methods: Resting-state magnetoencephalography was performed in 20 patients with glioma and 20 healthy subjects. The clustering coefficient of the resting functional connectivity network in the brain, average path length, and "small world" index (SWI) were calculated. Cognitive function was estimated by testing of attention, verbal fluency, memory, athletic ability, visualspatial ability, and intelligence.

Results: Compared with healthy controls, patients with glioma showed decreased cognitive function, and diminished low and high gamma band "small world" characteristics in the resting functional connectivity network.

Conclusion: The SWI is associated with cognitive function and is diminished in patients with glioma, and is therefore correlated with cognition dysfunction.

Keywords: glioma, cognitive dysfunction, "small world", functional connectivity network, magnetoencephalography

\section{Introduction}

Glioma patients often demonstrate cognitive dysfunction, including deficits in language, attention, and memory, which cannot be fully explained by local brain trauma. Given that cognition requires simultaneous participation of different brain regions, ${ }^{2}$ it is possible that disruption of the integrity of the brain contributes to its disrupted function. Evaluation of cognitive ability in patients with glioma is a critical part of the presurgical workup, but most evaluation scales used currently are subjective and can be affected by a number of factors, including the patient's educational background or general health status.

Magnetoencephalography (MEG) is a newly developed technique for analysis of the functional connectivity network of the brain, with better spatial and temporal resolution than positron emission tomography, electroencephalography, and functional magnetic resonance imaging (only temporally). ${ }^{4} \mathrm{MEG}$ maps brain activity by measuring changes in the magnetic field produced by electrical currents in the brain. Whether MEG-based analysis of brain connectivity can help with the clinical diagnosis of brain injury or tumor development is less well studied. The present study analyzed the resting-state functional connectivity network of the brain using MEG in 20 patients with glioma and 20 healthy controls. The results would provide general clinical implications in evaluating the cognitive function of glioma patients in an improved manner. 


\section{Materials and methods Clinical data}

MEG data were obtained for 20 patients with glioma (ten males and ten females, average age $38.85 \pm 2.77$ years, 19 righthanded, one left-handed) prior to surgery. All patients were confirmed as having glioma by pathological examination after surgery. Thirteen patients had World Health Organization (WHO) glioma grade I-II and seven patients had glioma WHO grade III-IV. Nine cases had glioma affecting the right hemisphere and 11 had glioma affecting the left hemisphere. The frontal lobe was involved in 10 cases, the temporal lobe in five, the parietal lobe in one, the frontoparietal area in two, the frontotemporal area in one, and the temporooccipital area in one. None of the patients had received radiotherapy or chemotherapy in the six months prior to MEG.

Twenty healthy controls (11 males and nine females, average age $33.55 \pm 3.20$ years, all right-handed) were also recruited, and magnetic resonance imaging confirmed that no cranial lesions were present. None of the subjects had received radiotherapy or psychoactive medication in the six months prior to MEG (two subjects received radiotherapy 1.5 years ago). There was no difference in mean age or gender distribution between the patients and healthy controls $(P>0.05)$.

\section{Cognitive testing}

All subjects were tested for attention, verbal fluency, memory, motor function, visuospatial abilities, and intelligence. The intelligence test included mathematical calculations, numbers and symbols, map filling, and similarity identification. The data are presented as $\mathrm{Z}$ scores. The tests used were the Wechsler Adult Intelligence Scale, the Wechsler Memory Scale, and Adult Neuropsychology Scale.

\section{MEG data acquisition and analysis}

A CTF/275 channel whole-head MEG system (VSA MedTech, Coquitlam, BC, Canada) was used for acquisition of the MEG data. The subjects were scanned while resting with eyes closed. The coils were positioned at the nasion and the left and right pre-auricular, and a 275 channel superconducting quantum interference-based whole head sensor was used to measure the magnetic field perpendicular to the scalp. Head movement was limited to $5 \mathrm{~mm}$ to confirm the recording accuracy. The original MEG wave was recorded every 120 seconds, with a total of five recordings made for each subject.

The MEG wave was analyzed for delta $(1-4 \mathrm{~Hz})$, theta $(4-8 \mathrm{~Hz})$, alpha $(8-12 \mathrm{~Hz})$, beta $(12-30 \mathrm{~Hz})$, low gamma (30-45 Hz), and high gamma $(55-70 \mathrm{~Hz})$ bands. The $2 \log$ threshold and phase lag index were analyzed. The clustering coefficient (C) and path length (L) were then calculated to construct a functional connectivity network. For small world analysis, the $\mathrm{S}$ index $=\mathrm{CS} / \mathrm{LS}$. A dual-pass Butterworth IIR filter was used during building of the network.

\section{Statistical analysis}

The data were analyzed using the Statistical Package for the Social Sciences version 13.0 software (Chicago, IL, USA). The Mann-Whitney $U$ test was used to compare the functional connectivity network in the resting state between the two groups and the $\mathrm{Z}$ scores for cognitive function. The Spearman correlation coefficient was used to analyze the relationship between the $\mathrm{Z}$ score and the "small world" index (SWI) in the patients with glioma. The Chi-square test was used for comparison of the gender distribution between the two groups, and the independent-samples $t$-test was used for comparison of the mean age of the two groups. $P<0.05$ was considered to be statistically significant.

\section{Results \\ Cognitive function}

The $\mathrm{Z}$ scores for attention $(P=0.03)$, verbal fluency $(P=0.00)$, calculation $(P=0.007)$, numbers and symbols $(P=0.03)$, and map filling tests $(P=0.008)$ were significantly decreased in patients with glioma compared with healthy controls.

\section{"Small world" index}

In healthy controls, the SWI for the resting-state functional connectivity network showed a positive correlation with the results of cognitive function testing, including attention with the low gamma band $(P=0.049$, R-squared linear regression $=0.061)$, visual-spatial tasks with the low $(P=0.006, \mathrm{R}$-squared linear regression $=0.236)$ and high $(P=0.04, \mathrm{R}$-squared linear regression $=0.23)$ gamma bands, calculation with the low $(P=0.04$, R-squared linear regression $=0.09)$ and high $(P=0.04$, R-squared linear regression $=0.17)$ gamma bands, map filling with the high gamma band $(P=0.023$, R-squared linear regression $=0.172$ ), and similarity testing with the alpha band $(P=0.04$, R-squared linear regression $=0.19)$ and low gamma band $(P=0.00, \mathrm{R}$-squared linear regression $=0.476$ ). No correlations were identified between verbal fluency, memory, motor ability, and SWI.

In patients with glioma, we found that the verbal fluency test was positively correlated with the high gamma band $(P=0.03, \mathrm{R}$-squared linear regression $=0.199)$, 
while the calculation test $(P=0.02$, R-squared linear regression $=0.241)$ and map filling test $(P=0.02$, R-squared linear regression $=0.263$ ) were positively correlated with the alpha band in the resting-state functional connectivity network.

\section{Discussion}

Previous studies have demonstrated that, in healthy adults, functional connectivity networks in the delta, theta, alpha, beta, low gamma, and high gamma bands show "small world" characteristics. ${ }^{5}$ Further, "small world" characteristics in the resting state are associated with synergy between brain regions and are positively correlated with cognitive function. ${ }^{6}$ The gamma band shows high specificity both spatially and temporally, and participates in associations across brain regions, ${ }^{7}$ and has also been linked with vision-based and hearing-based attention, ${ }^{8}$ as well as other types of cognitive function..$^{910}$

The present study has shown similar results. A positive correlation was found between brain function and specific bands. Unlike in healthy controls, small world network characteristics are lacking for the high and low gamma bands in patients with glioma. Such functional connectivity changes might herald underlying changes in general brain function and damage done by the tumor to local brain areas. It has been recognized that cognitive function can act as a diagnostic and prognostic factor in patients with glioma, even before radiological findings on computed tomography or magnetic resonance imaging. Currently, the evaluation scales and psychological tests available demonstrate limited application in actual clinical practice, because of the prohibitive costs involved and the difficulty in obtaining accurate measurements. It is believed that functional connectivity testing and identification of the "small world" characteristic might be used as a marker for cognitive dysfunction. Future studies are required to investigate the specificity of such changes in patients with glioma.

\section{Acknowledgments}

This study was supported by the grant from the clinical medical scientific and technologic project of Jiangsu province(BL2012041), and the grant from the medical scientific and technologic development project of Nanjing(YKK12138).

\section{Disclosure}

The authors report no conflicts of interest in this work.

\section{References}

1. Klein M, Heimans JJ, Boerman RH, et al. Effect of radiotherapy and other treatment related factors on mid-term to long-term cognitive sequelae in low-grade glioma: a comparative study. Lancet. 2002;360: 1361-1368.

2. Li Y, Liu Y, Li J, et al. Brain anatomical network and intelligence. PLoS Comput Biol. 2009;5:e1000395.

3. Chen Z, Wei D. Glioma-related cognitive dysfunction research progress Chin J Nerv Ment Dis. 2009;35:379-381.

4. Stevens MC. The developmental cognitive neuroscience of functional connectivity. Brain Cogn. 2009;70:1-12.

5. Lei T, Hu X, Yang K, et al. Functional connectivity network analysis of glioma by resting-state magnetoencephalography. Chin J of Stereotact Funct Neurosurg. 2011;24:326-330.

6. Douw L, Schoonheim MM, Landi D, et al. Cognition is related to resting-state small-world network topology: an magnetoencephalographic study. Neuroscience. 2011;175:169-177.

7. Kaiser J, Lutzenberger W. Induced gamma-band activity and human brain function. Neuroscientist. 2003;9:475-484.

8. Basar E, Basar-Eroglu C, Karakas S, et al. Gamma, alpha, delta, and theta oscillations govern cognitive processes. Int J Psychophysiol. 2001;39:241-248.

9. Herrmann CS, Frund I, Lenz D. Human gamma-band activity: a review on cognitive and behavioral correlates and network models. Neurosci Biobehav Rev. 2010;34:981-992.

10. Taphoorn MJ, Klein M. Cognitive deficits in adult patients with brain tumors. Lancet Neurol. 2004;3:159-168.
OncoTargets and Therapy

\section{Publish your work in this journal}

OncoTargets and Therapy is an international, peer-reviewed, open access journal focusing on the pathological basis of all cancers, potential targets for therapy and treatment protocols employed to improve the management of cancer patients. The journal also focuses on the impact of management programs and new therapeutic agents and protocols on

\section{Dovepress}

patient perspectives such as quality of life, adherence and satisfaction. The manuscript management system is completely online and includes a very quick and fair peer-review system, which is all easy to use. Visit http://www.dovepress.com/testimonials.php to read real quotes from published authors. 